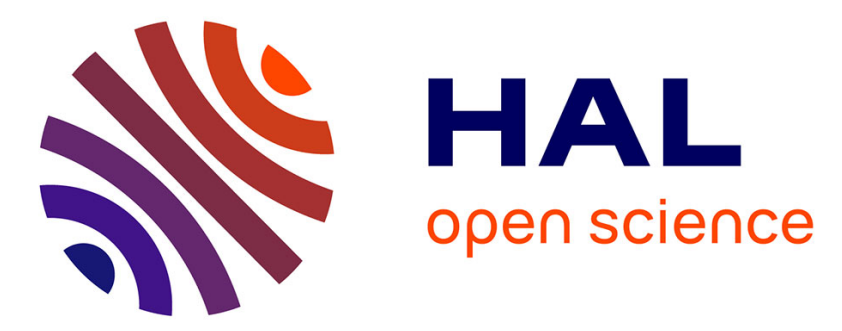

\title{
The electronic structure of the triiodide ion from relativistic correlated calculations: a comparison of different methodologies.
}

Andre Severo Pereira Gomes, Lucas Visscher, Hélène Bolvin, Trond Saue, Stefan Knecht, Timo Fleig, Ephraim Eliav

\section{To cite this version:}

Andre Severo Pereira Gomes, Lucas Visscher, Hélène Bolvin, Trond Saue, Stefan Knecht, et al.. The electronic structure of the triiodide ion from relativistic correlated calculations: a comparison of different methodologies.. Journal of Chemical Physics, 2010, 133 (6), pp.064305. 10.1063/1.3474571. hal-00760969

\section{HAL Id: hal-00760969 https://hal.science/hal-00760969}

Submitted on 21 Jan 2020

HAL is a multi-disciplinary open access archive for the deposit and dissemination of scientific research documents, whether they are published or not. The documents may come from teaching and research institutions in France or abroad, or from public or private research centers.
L'archive ouverte pluridisciplinaire HAL, est destinée au dépôt et à la diffusion de documents scientifiques de niveau recherche, publiés ou non, émanant des établissements d'enseignement et de recherche français ou étrangers, des laboratoires publics ou privés. 


\section{AIP|}

The electronic structure of the triiodide ion from relativistic correlated calculations: A

\section{comparison of different methodologies}

André Severo Pereira Gomes, Lucas Visscher, Hélène Bolvin, Trond Saue, Stefan Knecht, Timo Fleig, and Ephraim Eliav

Citation: The Journal of Chemical Physics 133, 064305 (2010); doi: 10.1063/1.3474571

View online: http://dx.doi.org/10.1063/1.3474571

View Table of Contents: http://scitation.aip.org/content/aip/journal/jcp/133/6?ver=pdfcov

Published by the AIP Publishing

\section{Articles you may be interested in}

First principles study of cobalt hydride, $\mathrm{CoH}$, and its ions $\mathrm{CoH}+$ and $\mathrm{CoH}-$

J. Chem. Phys. 137, 034309 (2012); 10.1063/1.4734595

Relativistic multireference calculation of photodissociation of o-, m-, and p-bromofluorobenzene

J. Chem. Phys. 134, 114303 (2011); 10.1063/1.3565445

Relativistic calculations of ground and excited states of LiYb molecule for ultracold photoassociation spectroscopy studies

J. Chem. Phys. 133, 124317 (2010); 10.1063/1.3475568

High-order electron-correlation methods with scalar relativistic and spin-orbit corrections

J. Chem. Phys. 126, 024104 (2007); 10.1063/1.2423005

Relativistic and correlated calculations on the ground, excited, and ionized states of iodine

J. Chem. Phys. 107, 9046 (1997); 10.1063/1.475194

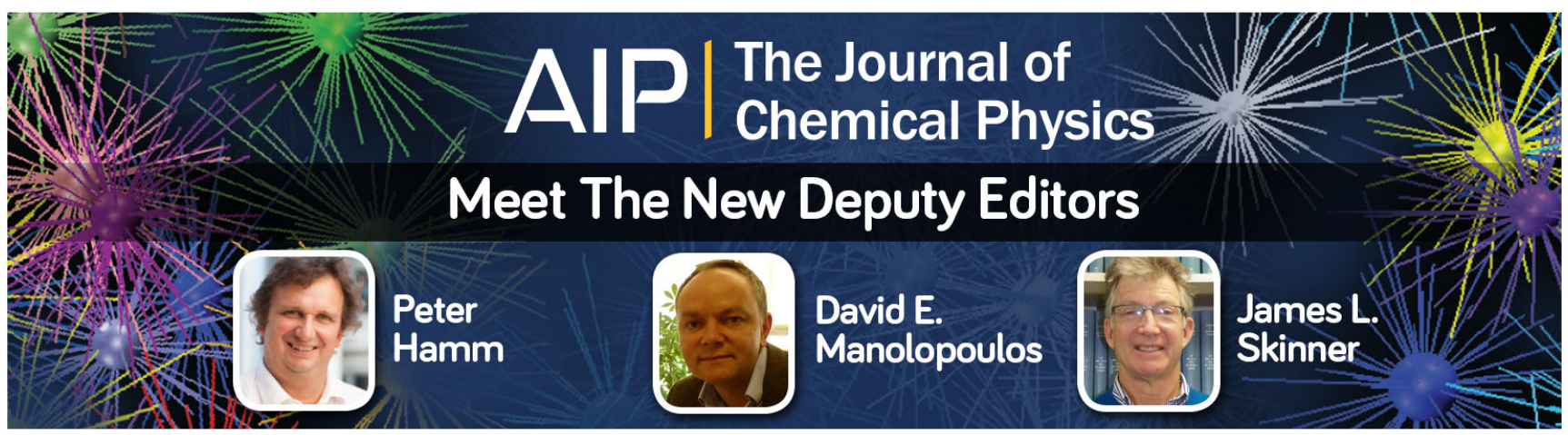




\title{
The electronic structure of the triiodide ion from relativistic correlated calculations: A comparison of different methodologies
}

\author{
André Severo Pereira Gomes, ${ }^{1,2,3}$ Lucas Visscher, ${ }^{2, a)}$ Hélène Bolvin, ${ }^{3}$ Trond Saue, $^{3}$ \\ Stefan Knecht, ${ }^{4,3}$ Timo Fleig, ${ }^{4,5}$ and Ephraim Eliav ${ }^{6}$ \\ ${ }_{1}^{1}$ Laboratoire PhLAM, CNRS UMR 8523, Université de Lille 1, F-59655 Villeneuve d'Ascq Cedex, France \\ ${ }^{2}$ Department of Theoretical Chemistry, Faculty of Sciences, Amsterdam Center for Multiscale \\ Modeling, VU University Amsterdam, De Boelelaan 1083, 1081 HV Amsterdam, The Netherlands \\ ${ }^{3}$ Laboratoire de Chimie Quantique, Institut de Chimie de Strasbourg, UMR 7177 CNRS/Université Louis \\ Pasteur, 4 rue Blaise Pascal, F-67000 Strasbourg, France \\ ${ }^{4}$ Institute of Theoretical and Computational Chemistry, Heinrich-Heine-University Duesseldorf, \\ Universitaetsstr. 1 D-40225 Duesseldorf, Germany \\ ${ }^{5}$ Laboratoire de Chimie et Physique Quantiques, I.R.S.A.M.C., Université Paul Sabatier, \\ 118 Route de Narbonne, F-31062 Toulouse, France \\ ${ }^{6}$ School of Chemistry, Tel Aviv University, 69978 Tel Aviv, Israel
}

(Received 30 March 2010; accepted 12 July 2010; published online 12 August 2010)

\begin{abstract}
The triiodide ion $\mathrm{I}_{3}^{-}$exhibits a complex photodissociation behavior, the dynamics of which are not yet fully understood. As a first step toward determining the full potential energy surfaces of this species for subsequent simulations of its dissociation processes, we investigate the performance of different electronic structure methods [time-dependent density functional theory, complete active space perturbation theory to second order (CASPT2), Fock-space coupled cluster and multireference configuration interaction] in describing the ground and excited states of the triiodide ion along the symmetrical dissociation path. All methods apart from CASPT2 include scalar relativity and spin-orbit coupling in the orbital optimization, providing useful benchmark data for the more common two-step approaches in which spin-orbit coupling is introduced in the configuration interaction. Time-dependent density functional theory with the statistical averaging of model orbital potential functional is off the mark for this system. Another choice of functional may improve performance with respect to vertical excitation energies and spectroscopic constants, but all functionals are likely to face instability problems away from the equilibrium region. The Fock-space coupled cluster method was shown to perform clearly best in regions not too far from equilibrium but is plagued by convergence problems toward the dissociation limit due to intruder states. CASPT2 shows good performance at significantly lower computational cost, but is quite sensitive to symmetry breaking. We furthermore observe spikes in the CASPT2 potential curves away from equilibrium, signaling intruder state problems that we were unable to curb through the use of level shifts. Multireference configuration interaction is, in principle, a viable option, but its computational cost in the present case prohibits use other than for benchmarking purposes. (C) 2010 American Institute of Physics. [doi:10.1063/1.3474571]
\end{abstract}

\section{INTRODUCTION}

Recent years have seen extraordinary advances in experimental techniques to probe chemical processes such as reaction dynamics in very short time frames. A wide range of techniques based on pump-probe schemes, ${ }^{1}$ where the species under consideration are set in a nonstationary state by one light source (pump) and monitored by another (probe), providing information regarding the dynamical behavior of the system. A particularly interesting field, where such fast techniques are very helpful, is the study of the dynamics of stable negative ions. ${ }^{2}$ By means of photodetachment or photodissociation these ions can be used to provide access to unstable neutral species that are difficult to study directly.

Some ionic species, however, are important in their own

\footnotetext{
a) Author to whom correspondence should be addressed. Electronic mail: visscher@chem.vu.nl.
}

right, apart from being used as precursors to other systems. A very well-known example is the triiodide ion $\left(\mathrm{I}_{3}^{-}\right)$. From a chemist's point of view, ${ }^{3-6}$ this relatively simple system is very interesting as it is (a) a structural analog of a transition state in $\mathrm{S}_{N} 2$ reactions and (b) an example of hyperconjugation, with a three-center four-electron bond. The widely accepted bonding picture of $\mathrm{I}_{3}^{-}$is that of a $(\sigma, \pi)$ system arising from the combinations of $5 \mathrm{p}$ orbitals on the three iodine atoms. In $\Lambda S$-coupling the ground state configuration is accordingly $\sigma_{u}^{2} \pi_{u}^{4} \pi_{g}^{4} \pi_{u}^{* 4} \sigma_{g}^{2}$, where the LUMO is the antibonding $\sigma_{u}^{*}$. This bonding picture is modified by spin-orbit coupling (SOC) (cf. Fig. 1), in particular for the MOs of gerade symmetry that are nonbonding and retain the atomic SO-mixing. In ungerade symmetry spin-orbit coupling is quenched due to bond formation and the orbitals are therefore essentially spin-pure. We have chosen to retain the $\Lambda S$ notation of all orbitals for easier reference to other work, but note that the MOs denoted $\sigma_{1 / 2 g}$ and $\pi_{1 / 2 g}$ in Fig. 1 corre- 


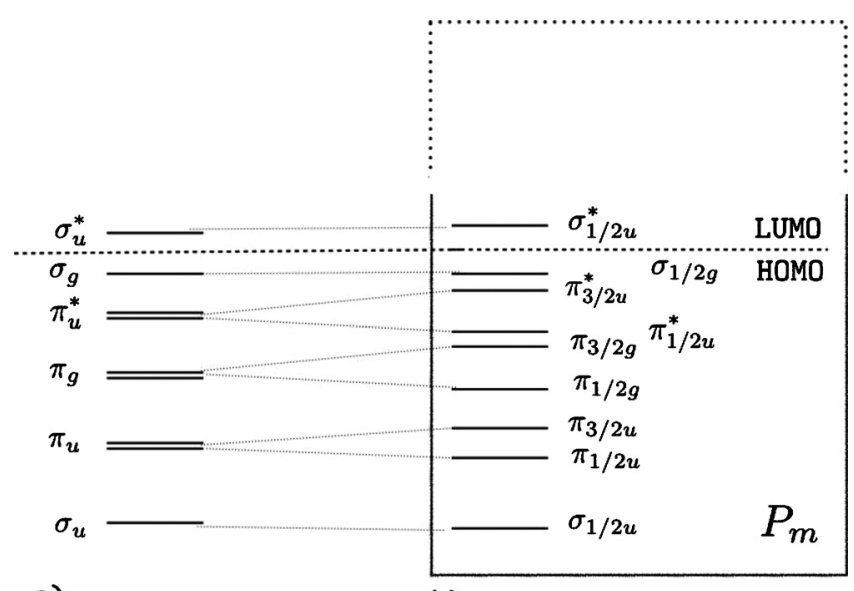

a)

b)

FIG. 1. Diagram of valence molecular orbitals of the triiodide ion, arising predominantly from the $5 \mathrm{p}$ manifold of the three iodine atoms: (a) spin-orbit free case and (b) spin-orbit case with the model $P_{m}$ space employed in the IHFSCC calculations indicated.

spond to linear combinations of $\mathrm{p}_{1 / 2}$ and $\mathrm{p}_{3 / 2}$ on the terminal atoms, respectively.

While early experimental information on the electronic structure of $\mathrm{I}_{3}^{-}$was obtained from UV spectroscopy, ${ }^{7}$ more detailed information has been gained from photodissociation studies in more recent works. These studies have originally been performed in solution. ${ }^{8-13}$ Questions arose as to whether it would be possible for the system to display a bent configuration at equilibrium, instead of the linear one proposed initially (the latter being the most accepted configuration). By contrast, experiments on the system in the gasphase by Neumark and co-workers ${ }^{14-16}$ and by Nagata and co-workers ${ }^{17,18}$ strongly suggest that the ground state for this species is linear and centrosymmetric. Photofragment yield (PFY) spectra ${ }^{15,17}$ reveal two photoabsorption bands peaking at 3.43-3.45 and 4.25-4.28 eV. They correspond to the $\mathrm{C}$ and D bands observed in solution, ${ }^{7}$ and the peak values can be assigned as the vertical excitation energies from the ground to the excited ${ }^{3} \Pi_{u}\left(0_{u}^{+}\right)$and ${ }^{1} \Sigma_{u}\left(0_{u}^{+}\right)$states. Time of flight photofragment mass spectrometry by Nakanishi et al. ${ }^{18}$ reveal a 40:60 ratio between three- and two-body dissociations both in the $\mathrm{C}$ and $\mathrm{D}$ bands. The three-body dissociation is dominated by charge-asymmetric dissociation that is with the negative charge on one of the terminal iodine atoms. The two-body dissociation in the $\mathrm{C}$ and $\mathrm{D}$ bands is dominated by the $\left(\mathrm{I}^{-}+\mathrm{I}_{2}\right)$ and $\left(\mathrm{I}+\mathrm{I}_{2}^{-}\right)$channels, respectively. Although the same dissociation channels appear in the fast beam photofragment translation spectroscopy of Neumark and co-workers, ${ }^{16}$ there are some discrepancies, notably in the calculated branching fractions for the two- and three-body dissociations as well as the $\left(\mathrm{I}^{-}+\mathrm{I}_{2}\right) /\left(\mathrm{I}+\mathrm{I}_{2}^{-}\right)$ratio, which call for further investigation.

Compared to the wealth of experimental data on the triiodide species, relatively few theoretical studies have been performed. Kosloff and co-workers, ${ }^{19}$ at about the time of the gas-phase experiments by Neumark, ${ }^{14,15}$ presented an extensive study of the potential energy curves of the $\mathrm{I}_{3}^{-}, \mathrm{I}_{2}$, and $\mathrm{I}_{2}^{-}$ species, which are thought to be involved in the photodissociation dynamics of $\mathrm{I}_{3}^{-}$. Their results, obtained with a combi- nation of multireference configuration interaction (MRCI) calculations and a diatomics-in-molecule (DIM) treatment where SOC effects were included, were the most accurate published at the time. However, the authors themselves considered the basis set used in the DIM treatment relatively small allowing for quantitative improvements on this early study. More recently, as a complement to their experimental work, Nakanishi et al. ${ }^{18}$ performed comprehensive spin-orbit configuration interaction (SOCI) calculations which explored potential surfaces not only along the symmetric stretch of $\mathrm{I}_{3}^{-}$ but also along the asymmetric stretch as well. Given the limited treatment of electron correlation and the still rather modest basis set size, also this theoretical investigation still leaves room for improvement.

Accordingly, the theoretical studies performed so far, although very helpful in understanding some aspects of the experimental data (such as the kind of states involved in the two experimentally observed absorption bands), are probably not sufficiently accurate for a direct comparison with experiment in terms of quantities associated with the dynamical behavior of the system, such as branching ratios. To enable this kind of analysis, it should be possible to calculate the whole of the potential energy surfaces accurately. This has motivated us to investigate the use of different methodologies for describing the electronic structure of the triiodide species to find the best candidate for calculation of a faithful representation of the potential energy surfaces that can be used in studies of the dynamics of the system.

Even though $\mathrm{I}_{3}^{-}$itself is a closed-shell species and can undergo two-body dissociation into two closed-shell species as well, it is important to account for SOC to achieve an accurate description of the full potential energy surfaces. Other possible channels involve dissociation into radicals, and furthermore SOC can have a significant impact on transition probabilities and intersystem crossings in the excitation/de-excitation processes. Apart from including SOC, theoretical methods should be able to describe the full potential energy surface (PES) and account for dynamical electron correlation at a reasonable cost, given the amount of calculations involved in determining a full PES.

As not all methods will fit this description we have chosen to compare expensive but accurate multireference coupled cluster (CC) and configuration interactions (CI) at selected points along the symmetric stretch of the molecule to methods that will allow full coverage of the ground and excited state potential energy surfaces. The wave functionbased methods employed were (a) the intermediate Hamiltonian Fock-space coupled cluster method of Eliav and co-workers, ${ }^{20-22}$ (b) the relativistic multireference CI method of Fleig and co-workers, ${ }^{23,24}$ and (c) the spin-orbit complete active space (CAS) perturbation theory to second order (PT2) method (SO-CASPT2) of Roos and co-workers. ${ }^{25,26}$ Apart from these, we decided to also explore the very economical time-dependent DFT (TDDFT) method ${ }^{27}$ to provide a simple orbital picture for the excitation processes. These TDDFT calculations are of course not expected to yield better results than any of the methods above due to the wellknown shortcomings of the current-day functionals (as, for 
instance, in describing charge-transfer excitations ${ }^{28,29}$ or that, within the adiabatic approximation, TDDFT can only describe single excitations ${ }^{30-32}$ ).

The paper is organized as follows: In Sec. II we present the details of the calculations performed with the different methodologies; in Sec. III we compare the performance of the different methods in calculating excitation energies of $\mathrm{I}_{3}^{-}$, both at selected bond lengths and at the equilibrium geometries for the symmetric configuration. We also take the opportunity to discuss results for the triiodide radical $\left(\mathrm{I}_{3}\right)$, a species that was experimentally observed in photoionization studies involving $\mathrm{I}_{3}^{-}$in the gas-phase, ${ }^{33,34}$ and that was also investigated theoretically by Kosloff and co-workers. Finally, in Sec. IV we assess the relative strengths and weaknesses of each method and provide concluding remarks.

\section{COMPUTATIONAL DETAILS}

All calculations described were performed along the symmetric stretch coordinate, with I-I bond lengths $r_{\mathrm{I}-\mathrm{I}}$ in the range of [2.60:6.00] $\AA$. In order to compare the different methodologies we have chosen two geometries in which to calculate the vertical excitation energies, apart from those obtained at the equilibrium structures for the different methods. These geometries are $r=2.84$ and $2.93 \AA$, corresponding to bond lengths in the vicinity of the equilibrium geometries for $\mathrm{I}_{3}$ (from the MRCI calculations of Kosloff and co-workers ${ }^{19}$ ) and $\mathrm{I}_{3}^{-}$(equilibrium geometry reported from solid state studies ${ }^{35}$ ), respectively.

\section{A. Intermediate Hamiltonian Fock-space coupled cluster}

Intermediate Hamiltonian Fock-space coupled cluster $^{20-22}$ (IHFSCC) calculations were performed with a development version of the DIRAC (Ref. 36) program. For describing the spectrum of $\mathrm{I}_{3}^{-}$with the IHFSCC method we have taken the anion as a starting point, and proceeded from the ground state through the $(1 h, 0 p)$ and $(0 h, 1 p)$ sectors in order to arrive at the $(1 h, 1 p)$ sector and, therefore, at the excitation energies,

$$
\mathrm{I}_{3}^{-}(0 h, 0 p) \rightarrow\left\{\mathrm{I}_{3}(1 h, 0 p), \mathrm{I}_{3}^{2-}(0 h, 1 p)\right\} \rightarrow \mathrm{I}_{3}^{-(*)}(1 h, 1 p) .
$$

For reasons of computational efficiency, the exact twocomponent Hamiltonian (X2C) scheme of Iliaš and Saue ${ }^{37}$ was used. Two-electron spin same-orbit (SSO) and spinother orbit (SOO) contributions were included via atomic mean-field integrals obtained with the $\mathrm{AMFI}^{38,39}$ code.

The triple zeta basis sets of Dyall ${ }^{40,41}$ were employed in all calculations. The starting large component $28 \mathrm{~s} 21 \mathrm{p} 15 \mathrm{~d}$ SCF set was kept uncontracted and augmented by $1 \mathrm{~s} 1 \mathrm{p} 1 \mathrm{~d} 1 \mathrm{f}$ diffuse functions. It thus contains the $2 \mathrm{~s} 2 \mathrm{p} 2 \mathrm{~d}$ primitives recommended for polarization and valence correlation as well as the $2 \mathrm{~s} 2 \mathrm{p} 2 \mathrm{~d}$ primitives recommended for core-valence correlation. By further augmentation two different sets were defined: a valence correlation set ("aVTZ"), where one correlating f-type function was added to the augmented SCF set, yielding a $(29 \mathrm{~s} 22 \mathrm{p} 16 \mathrm{~d} 2 \mathrm{f})$ basis set, and a core-valence correlating set ("aCVTZ"), which is a superset of aVTZ with additional $2 \mathrm{f} 1 \mathrm{~g}$ set of functions added.

In combination with these two sets different correlation spaces were employed: the first $\left(Q_{1}\right)$ is used together with basis set aVTZ and includes the orbitals with orbital energies $(\varepsilon)$ between -1 and 4 hartree. This means that in the occupied orbital space the $\sigma, \pi$ bonding system and three other $\sigma$-type orbitals arising from the $5 \mathrm{~s} 5 \mathrm{p}$ orbitals of iodine are present. The second correlation space $\left(Q_{2}\right)$ is used together with basis set aCVTZ and includes the orbitals with orbital energies between -3 and 12 hartree. This corresponds to enlarging $Q_{1}$ by including the occupied $4 \mathrm{~d}$ electrons of iodine apart from more virtual orbitals. The combinations aVTZ/ $Q_{1}$ and aCVTZ/ $Q_{2}$ will be referred to as IHFSCC(a) and $\operatorname{IHFSCC}(\mathrm{b})$, respectively.

A crucial ingredient of IHFSCC calculations to prevent convergence problems due to intruder states is the definition of the model $\left(P_{m}\right)$ and intermediate $\left(P_{I}\right)$ spaces that comprise the active space $P=P_{m}+P_{I \cdot}{ }^{21,22}$ After testing different spaces at the fixed geometries mentioned above, we found that convergence problems were generally avoided when the $P_{m}$ space contained 8 occupied $\left(1 \sigma_{g}, 1 \pi_{g}, 2 \pi_{u}\right.$, and $1 \sigma_{u}$ orbitals $)$ and 11 virtual orbitals $\left(2 \sigma_{g}, 1 \pi_{g}, 3 \sigma_{u}\right.$, and $2 \pi_{u}$ orbitals $)$ with a full $P$ space containing 11 occupied (5 of $g$ and 6 of $u$ parities) and 22 virtual (12 of $g$ and 10 of $u$ parities) orbitals (cf. Fig. 1). For bond lengths larger than $3.58 \AA$, however, it was not possible to obtain convergence for the $(1 h, 1 p)$ sector for this partition.

\section{B. $\mathrm{MRCl}$}

MRCI calculations have been carried out with the relativistic double group CI program LUCIAREL, ${ }^{23,24}$ which recently has been extended ${ }^{42,43}$ for parallel computer applications and to allow for the computation of molecular properties. $^{44}$ In all of the calculations reported here the aCVTZ basis set and the X2C Hamiltonian including twoelectron SSO and SOO corrections provided by the AMFI ${ }^{38,39}$ code have been employed.

The molecular spinors for the CI calculations have been obtained by an average-of-configuration Hartree-Fock calculation, where the open shells were defined as the 8 occupied Kramers pairs as in the CC application above, and in addition the antibonding $\sigma_{u}$ orbital, corresponding to an active subspace with 16 electrons in 9 Kramers pairs. This type of Hartree-Fock wave function comprises a good starting point for relativistic MRCI studies since it provides a balanced description of ground and electronically excited states. The concept of general active spaces has been employed for constructing the CI expansion. In the present case, all Slater determinants with zero, one, and two particles in the external space (truncated at 3 hartrees) were included and all possible active space distributions were allowed for the remaining electrons. These active space distributions were defined by a CAS space corresponding to the above space used in the average-of-configuration Hartree-Fock calculation [16 electrons in 9 Kramers pairs, or 15 electrons in 9 Kramers pairs in the case of neutral $\mathrm{I}_{3}$, "MRCI(a)"], and an additional space 
including the iodine $5 \mathrm{~s}$ orbitals where a restriction of up to two holes was imposed ["MRCI(b)"]. The resulting relativistic CI wave function describes the correlation of 16 (15 in the case of neutral $\mathrm{I}_{3}$ ) or 22 electrons and consists of roughly $77 \times 10^{6}$ Slater determinants in the latter case.

\section{CASPT2}

Calculations with the CASPT2 method were carried out with the MOLCAS code ${ }^{45}$ (version 7.0), within the CASSCF/ CASPT2/SO-RASSI approach. In this approach, scalar relativistic effects are included in the CASSCF (Ref. 25) and CASPT2 (Ref. 26) calculations via the Douglas-Kroll-Hess ${ }^{46}$ Hamiltonian, and in a subsequent calculation the CASPT2 spin-free states are used by the RASSI program to set up a spin-orbit coupling Hamiltonian. ${ }^{47}$ In this Hamiltonian the one- and two-electron spin-orbit integrals are calculated in a mean-field fashion via the $\mathrm{AMFI}^{38,39}$ code. The basis set used in these calculations was the ANO-RCC (Ref. 48) set of TZP quality (7s6p4d2f1g). All calculations were carried out in $C_{2 h}$ symmetry.

The CASSCF active space used consisted of 16 electrons in the nine orbitals arising from the $5 p$ orbitals of the three iodine atoms. We have kept the core orbitals (i.e., up to and including the $3 \mathrm{~d}$ orbitals) frozen in the CASSCF (and subsequent CASPT2) calculations. The CASSCF states that entered the multistate CASPT2 calculations have been obtained from state-averaged calculations over nine roots for ${ }^{1} \Sigma$, six roots for ${ }^{1} \Pi$, eight roots for ${ }^{3} \Sigma$, and six roots for ${ }^{3} \Pi$, respectively. In the CASPT2 calculations an IPEA shift of 0.25 a.u. was used. ${ }^{49}$

\section{TDDFT}

TDDFT calculations ${ }^{27}$ were carried out with the ADF (Ref. 50) code. Scalar relativistic and spin-orbit effects were included via the zeroth-order regular approximation. ${ }^{51}$ In the TDDFT calculations noncollinear spin magnetization was used. We are also within the adiabatic local density approximation (LDA) approximation to TDDFT, therefore disregarding any time-dependence of the exchange-correlation kernel, while using only the derivatives of the LDA functional for computing the exchange-correlation contributions to the excitation energies.

We used the statistical averaging of model orbital potentials (SAOPs) ${ }^{52}$ in combination with the TZ2P basis set. ${ }^{53}$ It should be noted that in the ADF implementation SAOP is used to construct the Kohn-Sham potential during the SCF cycle, whereas the ground state energy is calculated with the PW91 (Ref. 54) energy expression. This makes the ground state properties close to those obtained with PW91, the differences arising from the modification of the potential. More details can be found in the ADF documentation (for instance, Ref. 55). Given the dependence of TDDFT on the functional used, we shall use the label TDDFT/SAOP.

\section{RESULTS AND DISCUSSION}

In this section we discuss the results for the triiodide ion species obtained with the different methods. We begin by investigating the performance of the different methods in de-
TABLE I. Ground state spectroscopic constants $\left(r_{e}\right.$ in $\AA$ and $\omega_{e}$ in $\left.\mathrm{cm}^{-1}\right)$ of $\mathrm{I}_{3}^{-}$calculated with the DFT, MRCI(a), CASPT2, and $\operatorname{IHFSCC}(\mathrm{a}, \mathrm{b})$ methods.

\begin{tabular}{llc}
\hline \hline Method & $r_{e}$ & $\omega_{e}$ \\
\hline DFT & 3.007 & 102 \\
MRCI(a) & 2.982 & 108 \\
CASPT2 & 2.888 & 119 \\
IHFSCC(a) & 2.971 & 112 \\
IHFSCC(b) & 2.946 & 114 \\
MRCI (Ref. 19, spin-free) & 2.930 & 114 \\
DIM+SO (Ref. 19) & 2.966 & 95 \\
Expt. (Ref. 35) & 2.93 & $112 \pm 1$ \\
\hline \hline
\end{tabular}

termining ground state spectroscopic constants, before addressing the electronic spectrum. Given the exploratory nature of this paper, we will restrict ourselves to selected structures along the symmetric stretch coordinate.

When comparing methodologies, it should be kept in mind that each method, with the exception of TDDFT/SAOP, can be tuned through the selection of active electrons and orbitals. As reference we take IHFSCC(b), which we consider the most accurate methodology employed in this work.

We will focus mainly on vertical excitations calculated at selected geometries, but will also address "adiabatic" excitations for this particular cut of the surface, as a way to gain insight on how the different methods represent the overall shape of the surfaces. Finally, we will take a more detailed look the $0_{u}$ states, in particular, the absorbing $0_{u}^{+}$states, comparing the energetics and the excitation picture, in terms of the respective molecular orbitals for the different methods, before discussing the triiodide radical.

\section{A. Ground state spectroscopic constants for $I_{3}^{-}$}

The ground state spectroscopic constants of $\mathrm{I}_{3}^{-}$obtained here are shown in Table I. We take the experimental bond length ${ }^{35}$ in the solid state $\left(r_{e}=2.93 \AA\right)$ as a measure since to the best of our knowledge, there are no experimental bond length determinations for the triiodide ion in gas-phase.

With respect to this reference value CASPT2 shows a slight (about $0.04 \AA$ ) underestimation, whereas IHFSCC(a) shows a similar overestimation. Better agreement, with a deviation of less than $0.02 \AA$, is obtained with $\operatorname{IHFSCC}(b)$, where the $4 \mathrm{~d}$ shell is included in the occupied space and the virtual space is truncated at a higher energy. The effect of electron correlation can be studied in a more systematic manner at the CASPT2 level. Our default scheme is a CAS consisting of the $5 \mathrm{p}$ manifold and in addition correlating 4s4p4d5s at the PT2 level, giving a bond length of $2.888 \AA$. Freezing $4 \mathrm{~s} 4 \mathrm{p}$, which corresponds to the default CASPT2 correlation scheme in MOLCAS 7.2, increases the bond length to $2.914 \AA$ A Freezing $4 \mathrm{~d}$ gives an even more significant bond length extension to $2.966 \AA$, whereas in addition freezing $5 \mathrm{~s}$ shortens the bond slightly to $2.958 \AA$. These results suggest that the inclusion of the $4 \mathrm{~s} 4 \mathrm{p}$ shells in the occupied space of the IHFSCC calculations will bring the bond length in even closer agreement with experiment and thus implies that environmental effects on the bond length are small. We were unable to optimize the $\mathrm{I}_{3}^{-}$bond distance at the $\operatorname{MRCI}(\mathrm{b})$ level 
TABLE II. Comparison of vertical excitation energies $T_{v}$ (in eV) obtained with the different methods [TDDFT/SAOP, MRCI(a,b), CASPT2, and IHFSC$\mathrm{C}(\mathrm{a}, \mathrm{b})]$ for $\mathrm{I}_{3}^{-}$at $r_{1}=r_{2}=2.93 \AA$. States of the same symmetry as those for the optically active excited states are shown in boldface. Statistical measures of the error compared to IHFSCC(b) are also shown (see text for discussion).

\begin{tabular}{|c|c|c|c|c|c|c|c|c|c|c|c|c|}
\hline \multirow[b]{2}{*}{ State } & \multicolumn{2}{|c|}{ TDDFT/SAOP } & \multicolumn{2}{|c|}{ MRCI(a) } & \multicolumn{2}{|c|}{ MRCI(b) } & \multicolumn{2}{|c|}{ CASPT2 } & \multicolumn{2}{|c|}{ IHFSCC(a) } & \multicolumn{2}{|c|}{ IHFSCC(b) } \\
\hline & $\Omega$ & $T_{v}$ & $\Omega$ & $T_{v}$ & $\Omega$ & $T_{v}$ & $\Omega$ & $T_{v}$ & $\Omega$ & $T_{v}$ & $\Omega$ & $T_{v}$ \\
\hline 1 & $2 g$ & 1.92 & $2 g_{g}$ & 2.32 & $2 g_{g}$ & 2.30 & $2 g_{g}$ & 2.24 & $22_{g}$ & 2.10 & $2 g_{g}$ & 2.05 \\
\hline 2 & $1_{g}$ & 2.04 & $1_{g}$ & 2.44 & $0_{u}^{-}$ & 2.40 & $1_{g}$ & 2.32 & $1_{g}$ & 2.23 & $1_{g}$ & 2.18 \\
\hline 3 & $0_{u}^{-}$ & 2.42 & $0_{u}^{-}$ & 2.47 & $1_{u}$ & 2.41 & $0_{u}^{-}$ & 2.47 & $0_{u}^{-}$ & 2.26 & $0_{u}^{-}$ & 2.20 \\
\hline 4 & $1_{u}$ & 2.43 & $1_{u}$ & 2.48 & $1_{g}$ & 2.41 & $1_{u}$ & 2.47 & $1_{u}$ & 2.27 & $1_{u}$ & 2.20 \\
\hline 5 & $0_{g}^{-}$ & 2.50 & $0_{g}^{-}$ & 2.94 & $0_{g}^{-}$ & 2.91 & $0_{g}^{-}$ & 2.76 & $0_{g}^{-}$ & 2.68 & $0_{g}^{-}$ & 2.64 \\
\hline 6 & $0_{g}^{+}$ & 2.56 & $0_{g}^{+}$ & 2.98 & $0_{g}^{+}$ & 2.95 & $0_{g}^{+}$ & 2.82 & $0_{g}^{+}$ & 2.73 & $0_{g}^{+}$ & 2.69 \\
\hline 7 & $1_{g}$ & 2.70 & $1_{g}$ & 3.13 & $1_{g}$ & 3.08 & $1_{g}$ & 2.85 & $1_{g}$ & 2.90 & $1_{g}$ & 2.86 \\
\hline 8 & $2_{u}$ & 2.61 & $2_{u}$ & 3.25 & $2_{u}$ & 3.21 & $2_{u}$ & 3.10 & $2_{u}$ & 3.22 & $2_{u}$ & 3.17 \\
\hline 9 & $1_{u}$ & 2.72 & $1_{u}$ & 3.30 & $1_{u}$ & 3.25 & $1_{u}$ & 3.11 & $1_{u}$ & 3.30 & $1_{u}$ & 3.24 \\
\hline 10 & $\mathbf{0}_{\mathrm{u}}^{+}$ & 3.14 & $\mathbf{0}_{\mathrm{u}}^{+}$ & 3.71 & $\mathbf{0}_{\mathrm{u}}^{+}$ & 3.66 & $\mathbf{0}_{\mathrm{u}}^{+}$ & 3.52 & $\mathbf{0}_{\mathrm{u}}^{+}$ & 3.52 & $\mathbf{0}_{\mathrm{u}}^{+}$ & 3.51 \\
\hline 11 & $22_{g}$ & 3.50 & $0_{u}^{-}$ & 4.04 & $0_{u}^{-}$ & 3.98 & $0_{u}^{-}$ & 3.79 & $0_{u}^{-}$ & 3.95 & $2 g$ & 3.88 \\
\hline 12 & $0_{u}^{-}$ & 3.42 & $1_{u}$ & 4.09 & $1_{u}$ & 4.02 & $1_{u}$ & 3.80 & $2_{g}$ & 3.96 & $0_{u}^{-}$ & 3.91 \\
\hline 13 & $1_{g}$ & 3.63 & $2 g$ & 4.19 & $2_{g}$ & 4.16 & $2_{g}$ & 3.98 & $1_{u}$ & 4.03 & $1_{g}$ & 4.00 \\
\hline 14 & $1_{u}$ & 3.56 & $1_{g}$ & 4.29 & $1_{g}$ & 4.25 & $1_{g}$ & 4.06 & $1_{g}$ & 4.07 & $1_{u}$ & 4.00 \\
\hline 15 & $0_{g}^{-}$ & 4.10 & $0_{g}^{-}$ & 4.81 & $\mathbf{0}_{\mathrm{u}}^{+}$ & 4.75 & $\mathbf{0}_{\mathrm{u}}^{+}$ & 4.51 & $\mathbf{0}_{\mathrm{u}}^{+}$ & 4.33 & $\mathbf{0}_{\mathrm{u}}^{+}$ & 4.33 \\
\hline 16 & $0_{g}^{+}$ & 4.11 & $0_{g}^{+}$ & 4.82 & $0_{g}^{-}$ & 4.77 & $0_{g}^{-}$ & 4.51 & $0_{g}^{-}$ & 4.54 & $0_{g}^{-}$ & 4.48 \\
\hline 17 & $1_{g}$ & 4.34 & $\mathbf{0}_{\mathbf{u}}^{+}$ & 4.83 & $0_{g}^{+}$ & 4.78 & $0_{g}^{+}$ & 4.53 & $0_{g}^{+}$ & 4.54 & $0_{g}^{+}$ & 4.48 \\
\hline 18 & $\mathbf{0}_{\mathrm{u}}^{+}$ & 4.46 & $1_{g}$ & 4.96 & $1_{g}$ & 4.91 & $1_{g}$ & 4.60 & $2_{u}$ & 4.7 & $1_{g}$ & 4.68 \\
\hline $\bar{\Delta}$ & & -0.24 & & 0.25 & & 0.20 & & 0.05 & & 0.05 & & \\
\hline$\Delta_{\text {std }}$ & & 0.24 & & 0.11 & & 0.11 & & 0.15 & & 0.02 & & \\
\hline $\bar{\Delta}_{\mathrm{abs}}$ & & 0.31 & & 0.25 & & 0.21 & & 0.12 & & 0.05 & & \\
\hline$\Delta_{\max }$ & & 0.56 & & 0.50 & & 0.41 & & 0.27 & & 0.08 & & \\
\hline
\end{tabular}

due to computational constraints. Based on the trends observed with CASPT2, one would expect that $\mathrm{MRCI}(\mathrm{b})$, which correlates the $5 \mathrm{~s} 5 \mathrm{p}$, would overestimate the bond length further compared to $\mathrm{MRCI}(\mathrm{a})$. This is surprising since Vala et al. ${ }^{19}$ with the same active space and basis set quality reproduced the experimental bond length in their spin-orbit free MRCI calculations. One possible source of error in the present MRCI calculations is the truncation of the virtual space, but from previous experience we deem it unlikely that this truncation affects the bond length by $0.05 \AA$. We instead believe that the results of Vala et al. ${ }^{19}$ are fortuitous since our studies clearly show that correlation of the $4 \mathrm{~s} 4 \mathrm{p} 4 \mathrm{~d}$ manifold has a significant effect on the spectroscopic constants. Indeed, if we subtract the $0.07 \AA$ gained by correlating $4 \mathrm{~s} 4 \mathrm{p} 4 \mathrm{~d} 5 \mathrm{~s}$ at the CASPT2 level from our MRCI(a) result we come quite close to the experimental value.

It is interesting to note that DFT, where in principle all orbitals are correlated, overestimates $r_{e}$ by about $0.07 \AA$. Clearly, the SAOP potential was not derived with the aim of providing accurate ground state structures. On the other hand, other generalized gradient approximation (GGA) functionals do not perform better, e.g., PW91 (2.996 А), PBE

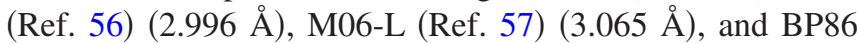
(3.007 $\AA$ ). The latter value contrasts significantly with the bond length of $3.14 \AA$ reported by Landrum et al., ${ }^{5}$ but they employed a TZP basis, thus not including the diffuse functions of the TZ2P basis as we do. Hybrid functionals do perform better, e.g., PBE0 (Ref. 58) (2.946 ^) and M06-2X (Ref. 59) (2.925 ̊), whereas LDA overbinds, giving $(2.913 \AA)$. We note in passing that the effect of spin-orbit coupling on the bond lengths is on the order of $0.02 \AA$ for the cited functionals, again an indication that the good agreement of the spin-orbit free MRCI calculation of Vala et $a l .{ }^{19}$ with the experimental bond length is likely to be fortuitous.

A similar picture is seen for the harmonic frequencies. Discrepancies between MRCI, CASPT2, IHFSCC, and both experiment $^{60}$ and the spin-free MRCI calculations of Kosloff are of the order of a few $\mathrm{cm}^{-1}$, whereas DFT underestimates the frequency by $10 \mathrm{~cm}^{-1}$. The spin-orbit numbers of Kosloff, on the other hand, show rather large discrepancies $\left(17 \mathrm{~cm}^{-1}\right)$, which may be due to artifacts arising from their diatomics-in-molecule treatment.

\section{B. Benchmark calculations of the electronic spectra of $\mathrm{I}_{3}^{-}$}

In the comparison of calculated spectra, we consider all methods both at the experimental bond length $(r=2.93 \AA)$ and at their own optimal ground state equilibrium geometries (shown in Table I). The corresponding spectra can be found in Tables II and III, respectively.

As already mentioned, we discuss the performance of the other methods relative to $\operatorname{IHFSCC}(b)$. This method is accurate for the first 18 excited states, which go up to about 4.5-5.0 eV, but becomes less trustworthy for higher energies due to the increasing importance of double excitations in these states. Double excitations are readily captured by MRCI and CASPT2 but neither by TDDFT/SAOP nor by the IHFSCC calculations on the $(1 h, 1 p)$ sector of Fock-space. 
TABLE III. Vertical $\left(T_{v}\right)$ excitation energies (in eV) calculated with the TDDFT/SAOP, MRCI(a), CASPT2, and IHFSCC(a,b) methods for I3 calculated at the optimum bond length for each method. States of the same symmetry as those for the optically active excited states are shown in boldface. Statistical measures of the error compared to IHFSCC(b) are also shown (see text for discussion).

\begin{tabular}{|c|c|c|c|c|c|c|c|c|c|c|}
\hline \multirow[b]{2}{*}{ State } & \multicolumn{2}{|c|}{ TDDFT/SAOP } & \multicolumn{2}{|c|}{ MRCI(a) } & \multicolumn{2}{|c|}{ CASPT2 } & \multicolumn{2}{|c|}{ IHFSCC(a) } & \multicolumn{2}{|c|}{$\operatorname{IHFSCC}(b)$} \\
\hline & $\Omega$ & $T_{v}$ & $\Omega$ & $T_{v}$ & $\Omega$ & $T_{v}$ & $\Omega$ & $T_{v}$ & $\Omega$ & $T_{v}$ \\
\hline 1 & $22_{g}$ & 1.67 & $22_{g}$ & 2.15 & $2_{g}$ & 2.38 & $2_{g}$ & 1.98 & $2_{g}$ & 2.00 \\
\hline 2 & $1_{g}$ & 1.78 & $1_{g}$ & 2.27 & $1_{g}$ & 2.45 & $1_{g}$ & 2.10 & $1_{g}$ & 2.13 \\
\hline 3 & $0_{u}^{-}$ & 2.13 & $0_{u}^{-}$ & 2.28 & $1_{u}^{s}$ & 2.63 & $0_{u}^{-}$ & 2.11 & $0_{u}^{-}$ & 2.13 \\
\hline 4 & $1_{u}$ & 2.14 & $1_{u}$ & 2.28 & $0_{u}^{-}$ & 2.63 & $1_{u}$ & 2.12 & $1_{u}$ & 2.14 \\
\hline 5 & $0_{g}^{-}$ & 2.24 & $0_{g}^{-}$ & 2.77 & $0_{g}^{-}$ & 2.90 & $0_{g}^{-}$ & 2.55 & $0_{g}^{-}$ & 2.58 \\
\hline 6 & $2_{u}^{8}$ & 2.26 & $0_{g}^{+}$ & 2.81 & $0_{g}^{+}$ & 2.95 & $0_{g}^{+}$ & 2.60 & $0_{g}^{+}$ & 2.64 \\
\hline 7 & $0_{g}^{+}$ & 2.31 & $1_{g}$ & 2.94 & $1_{g}^{g}$ & 3.00 & $1_{g}^{g}$ & 2.77 & $1_{g}^{g}$ & 2.81 \\
\hline 8 & $1_{u}^{g}$ & 2.38 & $2_{u}^{s}$ & 3.00 & $2_{u}^{\delta}$ & 3.31 & $2_{u}^{s}$ & 3.04 & $2_{u}^{\circ}$ & 3.10 \\
\hline 9 & $1_{g}$ & 2.44 & $1_{u}$ & 3.05 & $1_{u}$ & 3.32 & $1_{u}$ & 3.11 & $1_{u}$ & 3.17 \\
\hline 10 & $\mathbf{0}_{\mathbf{u}^{+}}^{+}$ & 2.82 & $\mathbf{0}_{\mathbf{u}}^{+}$ & 3.47 & $\mathbf{0}_{\mathrm{u}}^{+}$ & 3.72 & $\mathbf{0}_{\mathrm{u}}^{+}$ & 3.44 & $\mathbf{0}_{\mathrm{u}}^{+}$ & 3.36 \\
\hline 11 & $2 g$ & 3.07 & $0_{u}^{-}$ & 3.79 & $0_{u}^{-}$ & 3.99 & $2_{g}$ & 3.73 & $2_{g}$ & 3.79 \\
\hline 12 & $0_{u}^{-}$ & 3.09 & $1_{u}^{u}$ & 3.84 & $1_{u}^{u}$ & 4.00 & $0_{u}^{-}$ & 3.77 & $0_{u}^{-}$ & 3.84 \\
\hline 13 & $1_{g}$ & 3.20 & $2 "$ & 3.89 & $2_{g}$ & 4.23 & $1_{g}$ & 3.84 & $1_{g}$ & 3.90 \\
\hline 14 & $1_{u}$ & 3.22 & $1_{g}$ & 3.98 & $1_{g}$ & 4.31 & $1_{u}$ & 3.85 & $1_{u}$ & 3.93 \\
\hline 15 & $0_{g}^{-}$ & 3.66 & $0_{g}^{-}$ & 4.52 & $\mathbf{0}_{\mathbf{u}}^{+}$ & 4.67 & $\mathbf{0}_{\mathrm{u}}^{+}$ & 4.17 & $\mathbf{0}_{\mathrm{u}}^{+}$ & 4.27 \\
\hline 16 & $0_{g}^{+}$ & 3.68 & $0_{g}^{+}$ & 4.52 & $0_{g}^{-}$ & 4.75 & $0_{g}^{-}$ & 4.31 & $0_{g}^{-}$ & 4.38 \\
\hline 17 & $1_{g}$ & 3.91 & $\begin{array}{l}g \\
\mathbf{0}_{\mathbf{u}}^{+}\end{array}$ & 4.61 & $0_{g}^{g}$ & 4.78 & $0_{g}^{g}$ & 4.32 & $0_{g}^{+}$ & 4.39 \\
\hline 18 & $\mathbf{0}_{\mathbf{u}^{+}}^{+}$ & 4.29 & $1_{g}$ & 4.65 & $1_{g}^{8}$ & 4.85 & $1_{g}^{g}$ & 4.50 & $1_{g}^{8}$ & 4.59 \\
\hline $\bar{\Delta}$ & & -0.49 & & 0.09 & & 0.32 & & -0.05 & & \\
\hline$\Delta_{\text {std }}$ & & 0.29 & & 0.12 & & 0.12 & & 0.04 & & \\
\hline $\bar{\Delta}_{\mathrm{abs}}$ & & 0.49 & & 0.13 & & 0.32 & & 0.06 & & \\
\hline$\Delta_{\max }$ & & 0.84 & & 0.34 & & 0.50 & & 0.10 & & \\
\hline
\end{tabular}

In the statistical analysis of the errors for each method we therefore include only the first 18 states that are dominated by single excitations. In this analysis that is found at the bottom of Tables II and III, we report the mean error $\bar{\Delta}$ and its standard deviation $\Delta_{\text {std }}$, as well as the mean absolute error $\bar{\Delta}_{\text {abs }}$ and the maximum absolute error $\Delta_{\max }$ relative to the $\operatorname{IHFSCC}(b)$ results.

\section{Vertical excitations}

Inspecting Table II we immediately see some general trends: CASPT2 and MRCI tend to overestimate the IHFSCC excitation energies, whereas TDDFT/SAOP shows the lowest excitation energies among all methods considered. One can also see that the degree of electron correlation introduced by the different methods affects distinct regions of the spectrum differently. For the lowest ten excited states, the ordering of states is consistent with most methods whereas for higher states, where the density of states is higher, small variations in the correlation treatment result in significant reorderings. The IHFSCC(a) scheme yields only small deviations relative to the larger IHFSCC(b) calculation. This is quantified by the small mean errors (signed and absolute) and a small standard deviation $(0.02 \mathrm{eV})$, suggesting that core-valence correlation does not play a prominent role in describing the transitions to low-lying excited states. Such effects can be studied with more ease at the CASPT2 level. We find that freezing the $4 \mathrm{~s} 4 \mathrm{p}$ shells at the PT2 level has no effect on the vertical excitation energies, whereas freezing the $4 \mathrm{~d}$ shell brings about an upward shift of about $0.12 \mathrm{eV}$, somewhat larger than what is observed at the IHFSCC level.
Freezing in addition the $5 \mathrm{~s}$ shell leads to a mean upward shift of around $0.21 \mathrm{eV}$ compared to our default CASPT2 scheme. With these observations in mind one can readily understand why CASPT2 reproduces better the IHFSCC(b) reference than the MRCI calculations since the latter have much more limited active spaces. TDDFT/SAOP underestimates $(\bar{\Delta}=0.24 \mathrm{eV})$ the excitation energies, but perhaps more worrisome is that $\Delta_{\text {std }}$ is rather large $(0.24 \mathrm{eV})$, indicating large nonsystematic errors.

While it is beyond the scope of this paper to present a detailed analysis of the difference between IHFSCC and other methods, it is nevertheless instructive to consider comparisons of excitation energies calculated by IHFSCC and the linear response coupled cluster (LRCC) method done by various authors. ${ }^{61-65}$ From those studies it becomes clear that LRCC and IHFSCC may yield significantly different excitation energies. Some evidence from recent studies for different molecular systems performed by some of us ${ }^{66}$ as well as by other authors ${ }^{67-70}$ seems to point to a systematic upward shift in LRCC excitation energies compared to IHFSCC ones, similar to what is observed here when comparing MRCI and IHFSCC. This could be consistent with the different parametrization used in describing the excitations from the ground to the excited states (linear in MRCI and LRCC, and exponential for IHFSCC). It is less straightforward to rationalize the discrepancies between CASPT2 and IHFSCC as these methods describe the ground state with a rather different wave function. Based on previous experience $^{66,71-73}$ we expect CASPT2 results for low-lying 
excited states to deviate up to a few tenths of an $\mathrm{eV}$ from IHFSCC, but is more difficult to predict either an increase or a decrease of the excitation energies.

In Table III we present vertical excitation energies at the ground state equilibrium geometry optimized for each method. With a spread of $0.12 \AA$ between the optimized bond lengths, the discrepancies with respect to the reference $\operatorname{IHFSCC}(b)$ data increase. The effect is accentuated by the fact that the vertical excitation energies probe the repulsive wall of the excited states, as will become clear in Sec. III B 2. We first note that the vertical excitation energies obtained with the IHFSCC(b) scheme to the optically active states-3.36 and $4.27 \mathrm{eV}$ - are close to the experimentally observed peaks in the PFY spectra [3.43 and $4.25 \mathrm{eV}$ (Ref. 18)]. The agreement is also quite acceptable with the IHFSCC(a) scheme, which again indicates that core-valence correlation (correlating deeper than the $5 \mathrm{~s}$ shell) can possibly be disregarded when constructing the full potential energy surfaces. The corresponding CASPT2 results-3.72 and 4.67 $\mathrm{eV}$-are close to the SOCI values reported by Nakanishi et al. ${ }^{18}$ (3.79 and $\left.4.70 \mathrm{eV}\right)$. MRCI(a) is reasonably close to the lower excitation energy, but severely overestimates the second one, thus providing a peak separation energy of 1.14 $\mathrm{eV}$ which is far from the experimentally observed $0.82 \mathrm{eV}$. In fact, it is only the IHFSCC method that is able to reproduce the experimental peak positions and separation; all other methods explored in this paper overestimate the splitting significantly with TDDFT/SAOP being simply off the mark for these excitations.

The statistical analysis at these selected geometries indicates that both MRCI and CASPT2 are able to provide a balanced treatment of the ground and excited state surfaces. A problem with $\operatorname{MRCI}(\mathrm{a})$, currently the only feasible CI approach to cover the whole surface, is the fact that the optically active states (indicated in boldface in both Tables II and III) that are well described with IHFSCC(b) exhibit relatively large errors when treated with $\mathrm{MRCI}(\mathrm{a})$. An important remark concerning CASPT2 is that our conclusions are based on calculations carried out in $C_{2 h}$ symmetry with the rotation axis aligned with the molecular axis. Since we are presently only exploring the symmetric stretch one would rather be inclined to use the highest possible point group symmetry available for a centrosymmetric configuration, which is $D_{2 h}$ with the MOLCAS code. However, the introduction of a vertical mirror plane places $\pi_{x}$ and $\pi_{y}$ orbitals in different irreducible representations and introduces a symmetry breaking to which CASPT2 is extremely sensitive. Vertical excitation energies at bond length $2.93 \AA$ calculated in $D_{2 h}$ and $C_{2 v}$ symmetries give upper and lower bounds, respectively, for the corresponding $C_{2 h}$ values within a span approaching $0.1 \mathrm{eV}$. The choice of symmetry can therefore seriously affect conclusions regarding the performance of the method. On the other hand, restricting symmetry to $C_{2 h}$ means that the important distinction between $0^{+}$and $0^{-}$states is lost. The use of supersymmetry is hardly an option in the scan of the potential surfaces, so we have in the present work rather relied on scripts that analyze the CASSCF orbitals to extract this symmetry information. Having to work at re- duced symmetry also implies that orbitals are optimized and thus averaged for a larger number of states.

We also note that the two other experimentally observed ${ }^{7}$ bands - the A band (at $2.19 \mathrm{eV}$ in $\mathrm{CH}_{2} \mathrm{Cl}_{2}$ and $2.16 \mathrm{eV}$ in $\mathrm{Et}_{4} \mathrm{NI}_{3}$ crystal), assigned to a ${ }^{3} \Pi_{u}$, and the $\mathrm{B}$ band (at 2.82 $\mathrm{eV}$ in $\mathrm{CH}_{2} \mathrm{Cl}_{2}$ and $2.71 \mathrm{eV}$ in $\mathrm{Et}_{4} \mathrm{NI}_{3}$ crystal), assigned to a ${ }^{3} \Sigma_{u}^{+}$state - can indeed be correlated with the first and second $1_{u}$ states calculated for the vertical excitations with IHFSCC(b) (2.13 and $3.17 \mathrm{eV}$ ), IHFSCC(a) (2.11 and 3.11 $\mathrm{eV}$ ), MRCI(a) (2.28 eV and $3.05 \mathrm{eV}$ ), and TDDFT/SAOP (2.14 and $2.38 \mathrm{eV}$ ), while agreement with SO-CASPT2 is less good $(2.63$ and $3.32 \mathrm{eV})$. While the agreement for the maxima of band A is rather good, for band B it is much less so. This could be due to the fact that this band is rather weak and only visible as a shoulder on the $\mathrm{C}$ band in the spectra reported by Gabes and Stufkens, ${ }^{7}$ making the exact position dependent on the fitting procedure employed to establish the band maximum.

\section{Explorations of the dissociative region}

The discussion so far has dealt with geometries at and close to the ground state equilibrium structures. However, in order to be useful in modeling the dissociation process of $\mathrm{I}_{3}^{-}$ the potential surfaces of the excited states far from the equilibrium region have to be properly described as well. In order to probe the relative accuracy of the methods, we have chosen to investigate displacements along the symmetric stretch coordinate, along which photodissociation via the $\mathrm{C}$ and $\mathrm{D}$ bands generally initiates. This represents a one-dimensional cut through the full three-dimensional surface enabling us to define local minima (and harmonic frequencies) for the different excited states in this restricted geometry. One should thereby keep in mind, however, that such extrema do not necessarily correspond to the true spectroscopic constants for the corresponding states as we did not investigate the curvature of the surface in directions orthogonal to the symmetric stretch coordinate.

A further restriction in the current study is the presence of intruder states that made it impossible to converge the IHFSCC calculations at internuclear distances larger than 3.6 $\AA$. This means that we can only compare with the IHFSCC(b) reference at relatively short distances. Similarly, we could not obtain TDDFT/SAOP results beyond about $3.9 \AA$ due to triplet instabilities. In the CASPT2 calculations we observe downward spikes in the potential curves, visible in Fig. 2, appear around $3.5 \AA$, signaling a weak intruder state problem. These features appear already in the spin-orbit free CASPT2 calculations, whereas the CASSCF calculations produce smooth potential curves. We were unable to curb these features, applying real ${ }^{74}$ and imaginary ${ }^{75}$ level shifts of up to $0.20 E_{\mathrm{h}}$. On the other hand, outside the spikes the potential curves appear smooth and so a pragmatic approach to fitting the potential surfaces would be to remove points for which the weight of the reference CASSCF state drops below a selected value since this characterizes the appearance of the spikes. A more satisfying solution would be to increase the active space, but this easily brings the computational cost out of practical reach. 


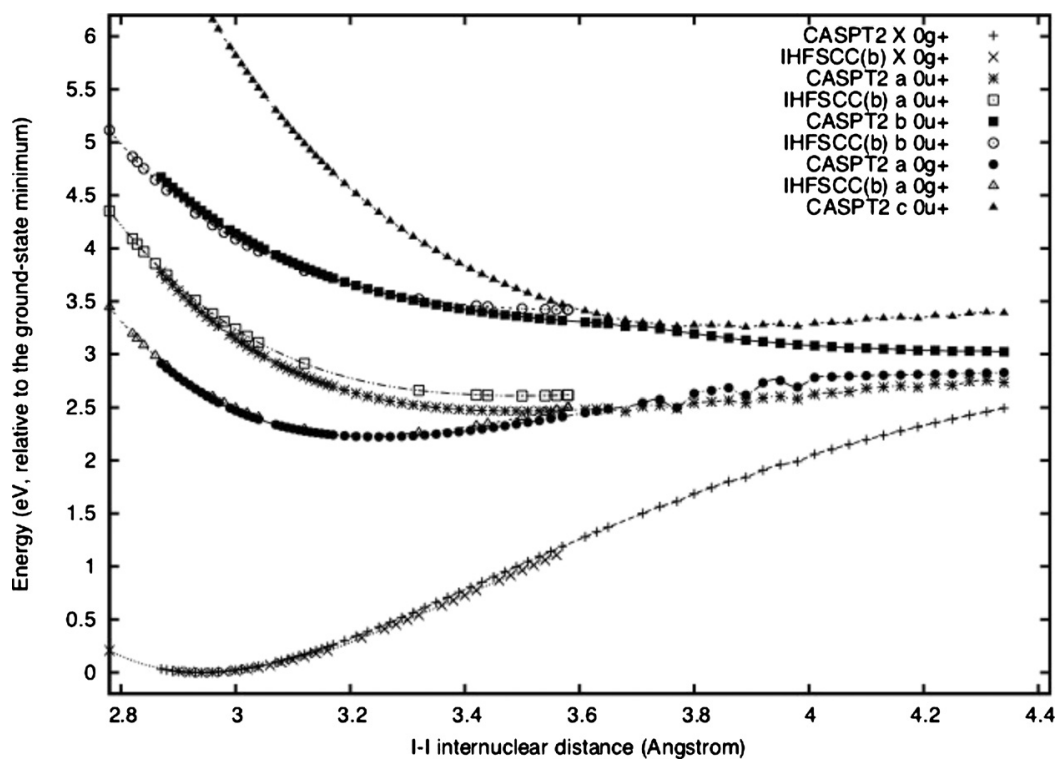

FIG. 2. Potential energy curves along the symmetric stretch coordinate for the ground state $\left(\mathrm{GS}, \mathrm{O}_{g}^{+}\right.$) and first excited $0_{g}^{+}$state, and lower $0_{u}^{+}$states (the first two corresponding to the optically active states) from CASPT2 and IHFSCC(b) calculations. All potentials were scaled so that the ground state minimum corresponds to the origin; the CASPT2 excited states were further shifted (a) downward by $0.31 \mathrm{eV}$ and (b) toward larger internuclear distances by $0.05 \AA$, to compensate the systematic errors compared to $\operatorname{IHFSCC}(b)$.

Potential curves along the symmetric stretch for the lower two $0_{g}^{+}$and three $0_{u}^{+}$states, calculated at the CASPT2 and IHFSCC(b) level, are given in Fig. 2. The crossing of the first excited $0_{g}^{+}$and $0_{u}^{+}$states around $3.7 \AA$ translate into a conical intersection in a 2-dimensional (2D) plot involving also the antisymmetric stretch and plays an important role in the photodissociation via the $\mathrm{C}$ band, as discussed by Nakanishi et al. ${ }^{18}$ These authors indicate a number of avoided crossings and conical intersections appearing in their SOCI calculations, such as an avoided crossing between the second and third $0_{u}^{+}$state, clearly visible around $3.7 \AA$ in Fig. 2, which renders the second $0_{u}^{+}$state, associated with the $\mathrm{D}$ band, dissociative. Due to these features in the potential curves we have restricted the calculation of spectroscopic constants and adiabatic excitation energies to the ten lowest excited states of $\mathrm{I}_{3}^{-}$, presented in Table IV.

The lower excited states are dominated by single excita- tions to the antibonding $\sigma_{1 / 2 u}^{*}$ LUMO and bond lengths are therefore systematically considerably longer than observed for the ground state. This also implies, as already mentioned, that vertical excitations probe the repulsive wall of the excited states. We note, for instance, that the vertical and adiabatic excitation energies to the first excited $0_{u}^{+}$, calculated at the IHFSCC(b) level, are 3.36 and $2.61 \mathrm{eV}$, respectively, a difference of $0.75 \mathrm{eV}$, meaning that the photodissociation process starts off with a significant acceleration of the vibrational wave packet.

With respect to the spectroscopic constants given in Table IV, we observe excellent agreement between IHFSCC(a) and IHFSCC(b), again suggesting that correlation of the $4 \mathrm{~d}$ shell is not crucial for a good description of the excited states potential surfaces. CASPT2 tends to consistently underestimate the bond lengths compared to $\operatorname{IHFSCC}(b)$ by about $0.07 \AA$, in line with the difference of $0.058 \AA$ for the

TABLE IV. Spectroscopic constants $\left(r_{e}\right.$ in $\AA$ and $\omega_{e}$ in $\left.\mathrm{cm}^{-1}\right)$ and adiabatic $\left(T_{e}\right)$ excitation energies (in eV) for the ten lowest excited states of $\mathrm{I}_{3}^{-}$, calculated with the TDDFT/SAOP, MRCI(a), CASPT2, IHFSCC(a), and IHFSCC(b) methods. The lowest, optically active $0_{u}^{+}$state is shown in boldface. Statistical measures of the error compared to IHFSCC(b) are also shown (see text for discussion). Values in parenthesis have reduced accuracy due to triplet instabilities (TDDFT) or lack of convergence (IHFSCC) and have not been included in the statistical analysis.

\begin{tabular}{|c|c|c|c|c|c|c|c|c|c|c|c|c|c|c|c|c|c|c|c|c|}
\hline \multirow[b]{2}{*}{ State } & \multicolumn{4}{|c|}{ TDDFT/SAOP } & \multicolumn{4}{|c|}{ MRCI(a) } & \multicolumn{4}{|c|}{ CASPT2 } & \multicolumn{4}{|c|}{ IHFSCC(a) } & \multicolumn{4}{|c|}{$\operatorname{IHFSCC}(\mathrm{b})$} \\
\hline & $\Omega$ & $r_{e}$ & $\omega_{e}$ & $T_{e}$ & $\Omega$ & $r_{e}$ & $\omega_{e}$ & $T_{e}$ & $\Omega$ & $r_{e}$ & $\omega_{e}$ & $T_{e}$ & $\Omega$ & $r_{e}$ & $\omega_{e}$ & $T_{e}$ & $\Omega$ & $r_{e}$ & $\omega_{e}$ & $T_{e}$ \\
\hline 1 & $2 g$ & 3.443 & 55 & 1.19 & $0_{u}^{-}$ & 3.501 & 56 & 1.53 & $22_{g}$ & 3.188 & 80 & 1.97 & $0_{u}^{-}$ & 3.439 & 60 & 1.45 & $0_{u}^{-}$ & 3.417 & 60 & 1.45 \\
\hline 2 & $1_{g}$ & 3.462 & 52 & 1.29 & $1_{u}$ & 3.503 & 56 & 1.53 & $1_{u}$ & 3.318 & 71 & 1.98 & $1_{u}$ & 3.436 & 61 & 1.46 & $1_{u}$ & 3.414 & 61 & 1.46 \\
\hline 3 & $0_{u}^{-}$ & (3.719) & $(22)$ & $(1.38)$ & $22_{g}$ & 3.383 & 61 & 1.64 & $0_{u}^{-}$ & 3.315 & 72 & 1.99 & $2 g_{g}$ & 3.270 & 81 & 1.59 & $2{ }_{g}$ & 3.245 & 83 & 1.59 \\
\hline 4 & $1_{u}$ & (3.696) & (29) & $(1.38)$ & $1_{g}$ & 3.421 & 56 & 1.71 & $1_{g}$ & 3.208 & 76 & 2.02 & $1_{g}$ & 3.282 & 78 & 1.70 & $1_{g}$ & 3.255 & 80 & 1.71 \\
\hline 5 & $2{ }_{u}$ & 3.616 & 46 & 1.41 & $2_{u}$ & 3.644 & 47 & 1.89 & $1_{u}$ & 3.427 & 59 & 2.33 & $0_{g}^{-}$ & 3.383 & 52 & 2.05 & $0_{g}^{-}$ & 3.358 & 53 & 2.07 \\
\hline 6 & $1_{u}$ & 3.669 & 39 & 1.52 & $1_{u}$ & 3.657 & 47 & 1.89 & $2{ }_{u}$ & 3.417 & 60 & 2.35 & $1_{g}$ & $\ldots$ & $\cdots$ & $\cdots$ & $1_{g}$ & (3.514) & (29) & $(2.20)$ \\
\hline 7 & $0_{g}^{+}$ & 3.388 & 63 & 1.68 & $0_{g}^{-}$ & 3.478 & 48 & 2.16 & $0_{g}^{-}$ & 3.222 & 73 & 2.44 & $2_{u}$ & 3.475 & 63 & 2.18 & $2_{u}$ & 3.446 & 64 & 2.22 \\
\hline 8 & $1_{g}$ & (3.877) & (54) & $(1.81)$ & $1_{g}$ & 3.562 & 43 & 2.22 & $1_{g}$ & 3.260 & 68 & 2.47 & $0_{g}^{+}$ & 3.264 & 82 & 2.22 & $0_{g}^{+}$ & 3.240 & 84 & 2.24 \\
\hline 9 & $0_{g}^{-}$ & 3.554 & 38 & 1.88 & $0_{g}^{+}$ & 3.388 & 62 & 2.28 & $0_{g}^{+}$ & 3.198 & 78 & 2.53 & $1_{u}$ & 3.492 & 62 & 2.21 & $1_{u}^{s}$ & 3.462 & 63 & 2.26 \\
\hline 10 & $\mathbf{0}_{\mathbf{u}}^{+}$ & 3.513 & 57 & 2.10 & $\mathbf{0}_{\mathbf{u}}^{+}$ & 3.731 & 34 & 2.36 & $\mathbf{0}_{\mathbf{u}}^{+}$ & 3.445 & 53 & 2.78 & $\mathbf{0}_{\mathrm{u}}^{+}$ & $\ldots$ & $\ldots$ & $\ldots$ & $\mathbf{0}_{\mathrm{u}}^{+}$ & 3.498 & 50 & 2.61 \\
\hline $\bar{\Delta}$ & & 0.163 & -18 & -0.52 & & 0.152 & -15 & -0.07 & & -0.066 & 3 & 0.31 & & 0.025 & -1 & -0.02 & & & & \\
\hline$\Delta_{\text {std }}$ & & 0.106 & 20 & 0.18 & & 0.051 & 8 & 0.19 & & 0.036 & 9 & 0.16 & & 0.003 & 1 & 0.02 & & & & \\
\hline $\bar{\Delta}_{\mathrm{abs}}$ & & 0.163 & 23 & 0.52 & & 0.152 & 15 & 0.14 & & 0.066 & 7 & 0.31 & & 0.025 & 1 & 0.02 & & & & \\
\hline$\Delta_{\max }$ & & 0.314 & -46 & -0.81 & & 0.233 & -24 & -0.37 & & -0.136 & 20 & 0.54 & & 0.030 & -2 & -0.05 & & & & \\
\hline
\end{tabular}


TABLE V. Character of $0_{u}^{+}$states of $\mathrm{I}_{3}^{-}$for TDDFT/SAOP, IHFSCC(b), CASPT2, and MRCI(a) at the respective ground state equilibrium geometries. These are given in terms of contributions from one-electron (for TDDFT, IHFSCC, and MRCI, where spin-orbit coupling is included at the SCF level) or many-electron states (for CASPT2). The oscillator strengths $\mathrm{f}$ associated to the transition from the ground state to these excited states are also shown (where available) along with the vertical excitation energy. The experimental absorption maxima are found at (a) 3.43 and $4.25 \mathrm{eV}$ from gas-phase photofragment yield spectra (Ref. 18), (b) 3.41 and $4.22 \mathrm{eV}$ in $\mathrm{CH}_{2} \mathrm{Cl}_{2}$ (solution) (Ref. 7), and (c) 3.38 and $4.28 \mathrm{eV}$ in $\mathrm{Et}_{4} \mathrm{NI}_{3}$ (solid state) (Ref. 7).

\begin{tabular}{|c|c|c|c|c|}
\hline Method & State & Excited state composition & $\begin{array}{c}T_{v} \\
(\mathrm{eV})\end{array}$ & f \\
\hline \multirow[t]{2}{*}{ TDDFT/SAOP } & $0_{u}^{+}$ & $51 \% \sigma_{1 / 2 g} \rightarrow \sigma_{1 / 2 u}^{*}+49 \% \pi_{1 / 2 g} \rightarrow \sigma_{1 / 2 u}^{*}$ & 2.82 & 0.09 \\
\hline & $0_{u}^{+}$ & $45 \% \sigma_{1 / 2 g} \rightarrow \sigma_{1 / 2 u}^{*}+50 \% \pi_{1 / 2 g} \rightarrow \sigma_{1 / 2 u}^{*}$ & 4.29 & 1.54 \\
\hline \multirow[t]{2}{*}{ MRCI } & $0_{u}^{+}$ & $46 \%_{1 / 2 g} \rightarrow \sigma_{1 / 2 u}^{*}+30 \% \pi_{1 / 2 g} \rightarrow \sigma_{1 / 2 u}^{*}+7 \%\left(\pi_{1 / 2 g}^{1} \pi_{1 / 2 u}^{1} \rightarrow \sigma_{1 / 2 u}^{* 2}\right)$ & 3.47 & 0.37 \\
\hline & $0_{u}^{+}$ & $29 \% \sigma_{1 / 2 g} \rightarrow \sigma_{1 / 2 u}^{*}+47 \% \pi_{1 / 2 g} \rightarrow \sigma_{1 / 2 u}^{*}+6 \%\left(\sigma_{1 / 2 g}^{1} \sigma_{1 / 2 u}^{1} \rightarrow \sigma_{1 / 2 u}^{* 2}\right)$ & 4.61 & 1.83 \\
\hline \multirow{3}{*}{ IHFSCC } & & $40 \% \sigma_{1 / 2 g} \rightarrow \sigma_{1 / 2 u}^{*}(\mathrm{LUMO})+19 \% \pi_{1 / 2 g} \rightarrow \sigma_{1 / 2 u}^{*}(\mathrm{LUMO})$ & & \\
\hline & $0_{u}^{+}$ & $+22 \% \sigma_{1 / 2 g} \rightarrow \sigma_{1 / 2 u}^{*}+15 \% \pi_{1 / 2 g} \rightarrow \sigma_{1 / 2 u}^{*}$ & 3.36 & $\mathrm{n} / \mathrm{a}^{\mathrm{a}}$ \\
\hline & $0_{u}^{+}$ & $\begin{aligned} 26 \% \sigma_{1 / 2 g} \rightarrow \sigma_{1 / 2 u}^{*}(\mathrm{LUMO})+36 \% \pi_{1 / 2 g} & \rightarrow \sigma_{1 / 2 u}^{*}(\mathrm{LUMO})+8 \% \sigma_{1 / 2 g} \\
\rightarrow \sigma_{1 / 2 u}^{*}+24 \% \pi_{1 / 2 g} & \rightarrow \sigma_{1 / 2 u}^{*}\end{aligned}$ & 4.27 & $\mathrm{n} / \mathrm{a}^{\mathrm{a}}$ \\
\hline \multirow[t]{2}{*}{ CASPT2 } & $0_{u}^{+}$ & $14 \%{ }^{1} \Sigma_{u}^{+}\left(98 \% \sigma_{g} \rightarrow \sigma_{u}^{*}\right)+85 \%{ }^{3} \Pi_{u}\left(91 \% \pi_{g} \rightarrow \sigma_{u}^{*}\right)$ & 3.72 & 0.45 \\
\hline & $0_{u}^{+}$ & $84 \%{ }^{1} \Sigma_{u}^{+}\left(98 \% \sigma_{g} \rightarrow \sigma_{u}^{*}\right)+15 \%{ }^{3} \Pi_{u}\left(91 \% \pi_{g} \rightarrow \sigma_{u}^{*}\right)$ & 4.67 & 2.37 \\
\hline
\end{tabular}

$\overline{\bar{a} \text { Not available. }}$

ground state. On the other hand, MRCI(a) significantly overestimates the excited state bond lengths, approaching the errors of TDDFT/SAOP $(\bar{\Delta} \simeq 0.16 \AA)$, although with lower standard deviation. The same tendency is observed for harmonic frequencies, where CASPT2 on the other hand shows rather good performance.

It is interesting then to note that MRCI(a) outperforms CASPT2 for adiabatic excitation energies, whereas TDDFT/ SAOP severely underestimates them. CASPT2 has, on the other hand, a reasonably small and consistent standard deviation of around $0.16 \mathrm{eV}$ in excitation energies. This systematic nature of the errors can therefore perhaps be exploited to further bring the results close to $\operatorname{IHFSCC(b)~by~applying~}$ global shifts to the CASPT2 potential energy surfaces. Such an approximation is demonstrated in Fig. 2, where we show, for the optically active $0_{u}^{+}$states, the $\operatorname{IHFSCC}(\mathrm{b})$ cuts of the potential energy surface along corresponding cuts of the CASPT2 potential, after the CASPT2 bond lengths were uniformly shifted by $0.035 \AA$ and a shift of $0.15 \mathrm{eV}$ was applied to the excited states. After these two corrections, we observe a very good agreement between the two methods, with rather small discrepancies for the second $0_{u}^{+}$state. Agreement for the first $0_{u}^{+}$state, however, seems to be somewhat poorer than for the second.

\section{A closer look at the $0_{u}^{+}$states}

The two strong, allowed transitions for $\mathrm{I}_{3}^{-}$occur from the $\Omega=0_{g}^{+}$ground state to two states with $\Omega=0_{u}^{+}$. Table $\mathrm{V}$ displays the dominant contributions to these transitions. If available we also show oscillator strengths for the corresponding transitions. Since the CASPT2 calculations were done in a two-step fashion we can also analyze the composition of these excited states in terms of spin-orbit free states. To a first approximation one can view these states as ${ }^{3} \Pi_{u}$ and ${ }^{1} \Sigma_{u}^{+}$, the former borrowing intensity from the latter through the spin-orbit coupling. This picture correlates very well with the DIM-SO results of Kosloff and co-workers. ${ }^{19}$ In an orbital picture all methods give the same description, with different mixings of the two transitions from the occupied $\sigma_{g}$ and $\pi_{g}$ orbitals to the $\sigma_{u}$ LUMO. In the IHFSCC(b) calculations two additional excitations appear that can be interpreted as providing orbital relaxation of the LUMO. Since the DFT calculations also include spin-orbit coupling at the SCF stage we see a similar picture as for IHFSCC and MRCI, except that relaxation effects do not play an important role (since the virtual orbitals see the same potential as the occupied ones).

Nakanishi et al. ${ }^{18}$ reported calculated squared transition moments $\left(\mu^{2}\right) 7.31$ and $13.51 a_{0}^{2}$ for the two lower $0_{u}^{+}$states, respectively, which translates into oscillator strengths of 0.68 and 1.56 , thus giving somewhat more relative weight to the lower state than what we observe at the CASPT2 and IHFSCC(b) level and shown in Table V. Gabes and Stufkens ${ }^{7}$ reported an intensity ratio of around 1.6:1.0 between the upper and lower $0_{u}^{+}$states. Since CASPT2, MRCI, and TDDFT/ SAOP all overestimate the peak separation between these states and thus underestimate the spin-orbit coupling between the underlying ${ }^{3} \Pi_{u}$ and ${ }^{1} \Sigma_{u}^{+} \Lambda S$ states, these methods are also unable to reproduce the experimental intensity ratio. IHFSCC does get the peak separation right, but unfortunately we do not have access to oscillator strengths for this methodology.

\section{Benchmark calculations on $\mathrm{I}_{3}$ : Electronic spectra and electron affinities}

Given that the IHFSCC results for the $(1 h, 0 p)$ sector are generated as by-products of the excitation energies determination for $\mathrm{I}_{3}^{-}$, we can also present spectroscopic constants and vertical and adiabatic excitation energies for this species, calculated with IHFSCC(b). We furthermore provide a comparison of this radical with IHFSCC, CASPT2, and MRCI at $r$ $=2.84 \AA$, which is the geometry employed by Kosloff and co-workers ${ }^{19}$ for the same system. These results are also shown in Table VI. 
TABLE VI. Comparison of excitation energies $T_{v}$ (in eV) for different methods [CASPT2, IHFSCC(a,b)], and MRCI(a) for neutral $\mathrm{I}_{3}$ at $r_{1}=r_{2}=2.84 \AA$ ] Vertical $\left(T_{v}\right)$ and adiabatic $\left(T_{e}\right)$ excitation energies, as well as EAs are shown for IHFSCC(b)

\begin{tabular}{|c|c|c|c|c|c|c|c|c|c|c|c|c|c|}
\hline \multirow[b]{2}{*}{ State $(\Omega)$} & \multirow[t]{2}{*}{$\mathrm{MRCI}^{\mathrm{a}}$} & MRCI(a) & \multirow[t]{2}{*}{ CASPT2 } & \multirow[t]{2}{*}{ IHFSCC(a) } & \multicolumn{3}{|c|}{ Expt. } & \multicolumn{6}{|c|}{ IHFSCC(b) } \\
\hline & & $T_{v}\left(r_{1}=\right.$ & & & $\omega_{e}$ & $T_{e}$ & $\mathrm{EA}^{\mathrm{b}}$ & $\mathrm{R}_{e}$ & $\omega_{e}$ & $T_{v}{ }^{\mathrm{c}}$ & $T_{v}$ & $T_{e}$ & $\mathrm{EA}^{\mathrm{d}}$ \\
\hline $\mathrm{X}\left(3 / 2_{u}\right)$ & 0.00 & 0.00 & 0.00 & 0.00 & $115 \pm 5$ & 0.00 & $4.15 \pm 0.12$ & 2.828 & 132 & 0.00 & 0.00 & 0.00 & 4.29 \\
\hline A $\left(1 / 2_{g}\right)$ & 0.28 & 0.33 & 0.42 & 0.27 & & 0.28 & & 2.884 & 113 & 0.24 & 0.25 & 0.23 & \\
\hline B $\left(1 / 2_{u}\right)$ & 0.61 & 0.65 & 0.55 & 0.64 & & 0.62 & & 2.837 & 129 & 0.65 & 0.65 & 0.65 & \\
\hline $\mathrm{C}\left(3 / 2_{g}\right)$ & 0.78 & 0.78 & 0.77 & 0.88 & & 0.68 & & 2.951 & 115 & 0.86 & 0.88 & 0.78 & \\
\hline $\mathrm{D}\left(1 / 2_{g}\right)$ & 1.62 & 1.63 & 1.55 & 1.68 & & & & 2.933 & 118 & 1.68 & 1.70 & 1.62 & \\
\hline
\end{tabular}

${ }^{\mathrm{a}}$ Results from Ref. 19 at $r_{1}=r_{2}=2.836 \AA$.

${ }^{\mathrm{b}}$ Reference 76; vertical detachment energy of $\mathrm{I}_{3}^{-}: 4.25 \mathrm{eV}$ (Ref. 34)

${ }^{\mathrm{c}}$ Calculated at $r_{1}=r_{2}=2.84 \AA$.

${ }^{\mathrm{d}}$ Value for the adiabatic EA. Vertical processes: $\operatorname{IP}\left(\mathrm{I}_{3}^{-}\right)=4.39 \mathrm{eV}$ and $\mathrm{EA}\left(\mathrm{I}_{3}\right)=4.20 \mathrm{eV}$.

The excitation energies calculated at $r=2.84 \AA$ are in good agreement with those of Kosloff et al., and, for the first two excited states, also with the experimental data. The $\Omega$ $=3 / 2_{g}$ third excited state has a much longer bond length than the ground state which may explain the $0.1-0.2 \mathrm{eV}$ overestimation found relative to the experimental value when calculated as vertical excitation. The adiabatic results for IHF$\mathrm{SCC}(\mathrm{b})$ indeed show a decrease of $0.10 \mathrm{eV}$ from the vertical to the adiabatic excitation energy.

In line with the discussion above, we see that the results obtained with the different methods are fairly consistent. We find a ground state with $\Omega=3 / 2_{u}$, followed by states of $\Omega$ $=1 / 2_{g}, 1 / 2_{u}, 3 / 2_{g}$, and $1 / 2_{g}$, respectively, in agreement with the experimental assignment. ${ }^{33,34}$

The CASPT2 excitation energies are lower than those of IHFSCC(b) by about $0.1-0.2 \mathrm{eV}$ with the exception of the first excited state, which is higher for CASPT2. In this case, however, MRCI and IHFSCC are much more alike than for $\mathrm{I}_{3}^{-}$, with discrepancies generally smaller than $0.1 \mathrm{eV}$. This strong similarity could be due to the fact that for the $(1 h, 0 p)$ sector used here the exponential parametrization of IHFSCC for the excited states is truncated at the linear term and therefore is essentially the same as in MRCI. ${ }^{62-65}$

From the IHFSCC(b) calculations we furthermore obtain an adiabatic (vertical) electron affinity (EA) of $4.29 \mathrm{eV}(4.20$ $\mathrm{eV}$ ) which compares well with the adiabatic EA of $4.15 \pm 0.12 \mathrm{eV}$, including zero-point vibrational corrections, obtained experimentally from a thermodynamic cycle, ${ }^{76}$ as well as the vertical detachment energy of $\mathrm{I}_{3}^{-}$of $4.25 \mathrm{eV}$ reported by Choi et $a .^{34}$

\section{CONCLUSIONS}

We have performed correlated electronic structure calculations including spin-orbit effects at high levels of theory on the triiodide ion $\left(\mathrm{I}_{3}^{-}\right)$and the radical $\left(\mathrm{I}_{3}\right)$. The agreement between the different wave function-based methodologies employed is reasonable, as is their agreement with experimental results. Exploratory TDDFT calculations with the SAOP functional provide a qualitatively correct picture not too far from the equilibrium distance, but show unsystematic errors that prohibit use in quantitative description. The SAOP functional was chosen on the one hand because it has been constructed to correct the wrong asymptotic behavior of pure exchange-correlation potentials, and on the other hand because it has shown good performance for excitation energies of molecules containing heavy elements. ${ }^{77-79}$ We believe that the principal reason for the relatively poor performance of TDDFT/SAOP is self-interaction errors. ${ }^{80}$ This is indicated by the severe overestimation of the equilibrium bond lengths and the observation that the introduction of exact Hartree-Fock exchange through hybrid functionals gives spectroscopic constants in much better agreement with experiment. We have not undertaken a systematic study of the performance of other DFT functionals. Whereas there is a good reason to believe that some functionals will provide better vertical excitation energies at the equilibrium distance, we suspect that they will all encounter stability problems along the dissociation channels.

With respect to $\mathrm{I}_{3}^{-}$, of the different methodologies evaluated, the intermediate Hamiltonian Fock-space coupled cluster is clearly the method that most accurately and consistently provides a picture which is both qualitatively and quantitatively correct for the excitation processes taking place in the initial steps in the photodissociation of the triiodide ion and the triiodide radical. Due to convergence problems this method is unfortunately not applicable to the complete potential energy surface, but we have been able to show that other wave function-based methods can reproduce the benchmark IHFSCC results rather well.

While it can be argued that for the triiodide species MRCI is slightly more accurate than SO-CASPT2, the latter has two interesting advantages: For one thing, it is computationally much more efficient than MRCI, and for another its errors seem to be very systematic for all electronic states considered. This systematic nature of errors is observed, in particular, for energies, allowing for global correction to be applied to the potential energy surfaces in order to bring them in agreement with IHFSCC. All is not well, however, with CASPT2. We observe a strong sensitivity of the method to symmetry breaking. A simulation of the photodissociation of $\mathrm{I}_{3}^{-}$will require as a minimum the generation of the $2 \mathrm{D}$ potential surfaces of linear $\mathrm{I}_{3}^{-}$. However, in order to avoid symmetry breaking such a scan can at best be carried out in $C_{2}$ symmetry, for which the distinction between $0^{+}$and $0^{-}$ states is lost and needs to be recovered by the use of scripts when analyzing the individual states. CASPT2 is furthermore susceptible to intruder states, and we have been unable 
to remove the spikes observed in the potential curves toward dissociation by the use of level shifting techniques. One alternative is to explore a possibly more robust multireference perturbation theory such as n-electron valence second-order perturbation theory. ${ }^{81}$

MRCI clearly is another option, but presently too expensive to be able to generate a full set of potential energy surfaces for studies of the dynamics of the photodissociation process. The challenge of applying such methods to heavyelement systems is not only the mandatory treatment of spinorbit coupling, but also the fact that heavy atoms are increasingly polarizable such that subvalence has to be correlated. In the present case we observe that the correlation of $4 d$ is crucial for obtaining correct spectroscopic constants, less so for vertical excitation energies. Subvalence correlation can possibly be avoided by employing relativistic effective core potentials combined with core-polarization potentials. ${ }^{82,83} \mathrm{Fi}-$ nally, we would like to suggest that a successful combination of MCSCF and DFT (Ref. 84) could be the ideal tool for generating potential surfaces for dynamics.

\section{ACKNOWLEDGMENTS}

We dedicate this paper to a giant of quantum chemistry, Björn Roos. Bravely fighting illness, he succumbed in the end. In the preparation of this work he (as well as Per-Åke Malmqvist and Valera Veryazov) was most helpful in answering questions related to the SO-CASPT2 calculations. Once, only a few days after a mail with questions, he answered as usually to the point, but excusing himself for the late reply. His mail was written an early Sunday morning, at a time when most of his younger and healthier colleagues were probably still sound asleep.

We wish to thank Professor Ronnie Kosloff for calling our attention to the need for a more accurate description of the potential energy surfaces for the triiodide dissociation problem. Moreover, we thank the DEISA Consortium (cofunded by the EU, FP6 Project No. 508830/031513), for support within the DEISA Extreme Computing Initiative (www.deisa.org). In particular, we wish to acknowledge the work done by Walter Lioen (SARA/NL) in improving the efficiency of the four-index transformation code within the DIRAC program for the architectures used. We also acknowledge support by CINES ("Centre Informatique National de l'Enseignement Supérieur") under Grant No. ph12531. This work has been supported by the Netherlands Organization for Scientific Research (NWO) via a Vici grant for L.V.

\footnotetext{
${ }^{1}$ M. Fushitani Annu. Rep. Prog. Chem., Sect. C: Phys. Chem. 104, 272 (2008).

${ }^{2}$ D. M. Neumark, J. Chem. Phys. 125, 132303 (2006).

${ }^{3}$ R. J. Hach and R. E. Rundle, J. Am. Chem. Soc. 73, 4321 (1951).

${ }^{4}$ G. C. Pimentel, J. Chem. Phys. 19, 446 (1951).

${ }^{5}$ G. A. Landrum, N. Goldberg, and R. Hoffmann, J. Chem. Soc. Dalton Trans. 1997, 3605.

${ }^{6}$ P. H. Svensson and L. Kloo, Chem. Rev. (Washington, D.C.) 103, 1649 (2003).

${ }^{7}$ W. Gabes and D. J. Stufkens, Spectrochim. Acta, Part A 30, 1835 (1974).

${ }^{8}$ M. Mizuno, J. Tanaka, and I. Harada, J. Phys. Chem. 85, 1789 (1981).

${ }^{9}$ H. Isci and W. R. Mason, Inorg. Chem. 24, 271 (1985).

${ }^{10}$ T. Kühne and P. Vöhringer, J. Chem. Phys. 105, 10788 (1996).
}

${ }^{11}$ T. Kühne, R. Küster, and P. Vöhringer, Chem. Phys. 233, 161 (1998).

${ }^{12}$ T. Kühne and P. Vöhringer, J. Phys. Chem. A 102, 4177 (1998).

${ }^{13}$ A. Baratz and S. Ruhman, Chem. Phys. Lett. 461, 211 (2008).

${ }^{14}$ M. T. Zanni, B. J. Greenblatt, A. V. Davis, and D. M. Neumark, J. Chem. Phys. 111, 2991 (1999)

${ }^{15}$ H. Choi, R. T. Bise, A. A. Hoops, and D. M. Neumark, J. Chem. Phys. 113, 2255 (2000)

${ }^{16}$ A. A. Hoops, J. R. Gascooke, A. E. Faulhaber, K. E. Kautzman, and D. M. Neumark, J. Chem. Phys. 120, 7901 (2004).

${ }^{17}$ L. Zhu, K. Takahashi, M. Saeki, T. Tsukuda, and T. Nagata, Chem. Phys. Lett. 350, 233 (2001).

${ }^{18}$ R. Nakanishi, N. Saitou, T. Ohno, S. Kowashi, S. Yabushita, and T. Nagata, J. Chem. Phys. 126, 204311 (2007).

${ }^{19}$ J. Vala, R. Kosloff, and J. N. Harvey, J. Chem. Phys. 114, 7413 (2001).

${ }^{20}$ L. Visscher, E. Eliav, and U. Kaldor, J. Chem. Phys. 115, 9720 (2001).

${ }^{21}$ A. Landau, E. Eliav, Y. Ishikawa, and U. Kaldor, J. Chem. Phys. 113, 9905 (2000).

${ }^{22}$ A. Landau, E. Eliav, Y. Ishikawa, and U. Kaldor, J. Chem. Phys. 115, 6862 (2001)

${ }^{23}$ T. Fleig, J. Olsen, and L. Visscher, J. Chem. Phys. 119, 2963 (2003).

${ }^{24}$ T. Fleig, H. J. Aa. Jensen, J. Olsen, and L. Visscher, J. Chem. Phys. 124, 104106 (2006).

${ }^{25}$ B. O. Roos, P. R. Taylor, and P. E. M. Siegbahn, Chem. Phys. 48, 157 (1980).

${ }^{26}$ K. Andersson, P.-A. Malmqvist, B. O. Roos, A. J. Sadlej, and K. Wolinski, J. Phys. Chem. 94, 5483 (1990).

${ }^{27}$ E. K. U. Gross and W. Kohn, Adv. Quantum Chem. 21, 255 (1990).

${ }^{28}$ D. Tozer, J. Chem. Phys. 119, 12697 (2003).

${ }^{29}$ M. E. Casida, F. Gutierrez, J. Guan, F.-X. Gadea, D. Salahub, and J.-P. Daudey, J. Chem. Phys. 113, 7062 (2000).

${ }^{30}$ M. E. Casida, J. Chem. Phys. 122, 054111 (2005).

${ }^{31}$ D. J. Tozer and N. C. Handy, Phys. Chem. Chem. Phys. 2, 2117 (2000).

${ }^{32}$ N. T. Maitra, F. Zhang, R. J. Cave, and K. Burke, J. Chem. Phys. 120, 5932 (2004).

${ }^{33}$ T. R. Taylor, K. R. Asmis, M. T. Zanni, and D. M. Neumark, J. Chem. Phys. 110, 7607 (1999).

${ }^{34}$ H. Choi, T. R. Taylor, R. R. Bise, A. A. Hoops, and D. M. Neumark, J. Chem. Phys. 113, 8608 (2000)

${ }^{35}$ T. Migchelsen and A. Vos, Acta Crystallogr. 23, 796 (1967).

${ }^{36}$ DIRAC, a relativistic ab initio electronic structure program, release DIRAC04.0, written by H. J. Aa. Jensen, T. Saue, and L. Visscher with contributions from V. Bakken, E. Eliav, T. Enevoldsen, T. Fleig, O. Fossgaard, T. Helgaker, J. Laerdahl, C. V. Larsen, P. Norman, J. Olsen, M. Pernpointner, J. K. Pedersen, K. Ruud, P. Salek, J. N. P. van Stralen, J. Thyssen, O. Visser, and T. Winther, 2004.

${ }^{37}$ M. Iliaš and T. Saue, J. Chem. Phys. 126, 064102 (2007).

${ }^{38}$ B. Schimmelpfennig, AMFI: an atomic mean-field code, Stockholm, Sweden, 1996.

${ }^{39}$ B. A. Heß, C. M. Marian, U. Wahlgren, and O. Gropen, Chem. Phys. Lett. 251, 365 (1996)

${ }^{40}$ K. G. Dyall, Theor. Chem. Acc. 108, 335 (2002).

${ }^{41}$ K. G. Dyall, Theor. Chem. Acc. 115, 441 (2006).

${ }^{42}$ S. Knecht, H. J. Aa. Jensen, and T. Fleig, J. Chem. Phys. 128, 014108 (2008).

${ }^{43}$ S. Knecht, H. J. Aa. Jensen, and T. Fleig, J. Chem. Phys. 132, 014108 (2010).

${ }^{44}$ S. R. Knecht, "Parallel relativistic multiconfiguration methods: New powerful tools for heavy-element electronic-structure studies," Ph.D. dissertation, Heinrich-Heine-Universität Düsseldorf, 2009.

${ }^{45}$ G. Karlström, R. Lindh, P.-A. Malmqvist, B. O. Roos, U. Ryde, V. Veryazov, P.-O. Widmark, M. Cossi, B. Schimmelpfennig, P. Neogrady, and L. Seijo, Comput. Mater. Sci. 28, 222 (2003).

${ }^{46}$ B. A. Hess, Phys. Rev. A 33, 3742 (1986)

${ }^{47}$ P.-A. Malmqvist, B. O. Roos, and B. Schimmelpfennig, Chem. Phys. Lett. 357, 230 (2002).

${ }^{48}$ B. O. Roos, R. Lindh, P.-A. Malmqvist, V. Veryazov, and P.-O. Widmark, J. Phys. Chem. A 108, 2851 (2004).

${ }^{49}$ G. Ghigo, B. O. Roos, and P.-A. Malmqvist, Chem. Phys. Lett. 396, 142 (2004).

${ }^{50}$ ADF, Amsterdam density functional program, Theoretical Chemistry, Vrije Universiteit Amsterdam, URL: http://www.scm.com, 2007.

${ }^{51}$ E. van Lenthe, J. G. Snijders, and E. J. Baerends, J. Chem. Phys. 105, 6505 (1996).

${ }^{52}$ P. R. T. Schipper, O. V. Gritsenko, S. J. A. van Gisbergen, and E. J. 
Baerends, J. Chem. Phys. 112, 1344 (2000).

${ }^{53}$ E. van Lenthe and E. J. Baerends, J. Comput. Chem. 24, 1142 (2003).

${ }^{54}$ J. Perdew, J. Chevary, S. Vosko, K. Jackson, M. Pederson, D. Sing, and C. Fiolhais, Phys. Rev. B 46, 6671 (1992).

${ }^{55}$ For further details on the use of the SAOP potential, see the ADF document http://www.scm.com/Doc/Doc2009.01/ADF/ADFUsersGuide/ page $95 . h t m l$, retrieved July 14, 2010.

${ }^{56}$ K. B. J. P. Perdew and M. Ernzerhof, Phys. Rev. Lett. 77, 3865 (1996)

${ }^{57}$ Y. Zhao and D. G. Truhlar, J. Chem. Phys. 125, 194101 (2006).

${ }^{58}$ C. Adamo and V. Barone, J. Chem. Phys. 110, 6158 (1999).

${ }^{59}$ Y. Zhao and D. G. Truhlar, Theor. Chem. Acc. 120, 215 (2008).

${ }^{60}$ M. T. Zanni, V. S. Batista, W. H. Miller, B. J. Greenblatt, A. V. Davis, and D. M. Neumark, J. Chem. Phys. 110, 3748 (1999).

${ }^{61}$ T. Helgaker, P. Jørgensen, and J. Olsen, Molecular Electronic Structure Theory (Wiley, Chichester, 2000).

${ }^{62}$ R. J. Bartlett and M. Musial, Rev. Mod. Phys. 79, 291 (2007).

${ }^{63}$ L. Meissner and R. J. Bartlett, J. Chem. Phys. 94, 6670 (1991).

${ }^{64}$ L. Meissner and R. J. Bartlett, J. Chem. Phys. 102, 7490 (1995).

${ }^{65}$ D. Mukhopadhyay, S. Mukhopadhyay, R. Chaudhuri, and D. Mukherjee, Theor. Chim. Acta 80, 441 (1991).

${ }^{66}$ F. Réal, A. S. P. Gomes, L. Visscher, V. Vallet, and E. Eliav, J. Phys. Chem. A 113, 12504 (2009).

${ }^{67}$ L. Meissner, J. Chem. Phys. 108, 9227 (1998).

${ }^{68}$ M. Musial and R. J. Bartlett, J. Chem. Phys. 129, 044101 (2008).

${ }^{69}$ M. Musial and R. J. Bartlett, J. Chem. Phys. 129, 134105 (2008).

${ }^{70}$ M. Musial and R. J. Bartlett, Chem. Phys. Lett. 457, 267 (2008).
${ }^{71}$ I. Infante, A. S. P. Gomes, and L. Visscher, J. Chem. Phys. 125, 074301 (2006).

${ }^{72}$ G. La Macchia, I. Infante, R. Juraj, J. K. Gibson, and L. Gagliardi, Phys. Chem. Chem. Phys. 10, 7278 (2008).

${ }^{73}$ F. Notter, S. Dubillard, and H. Bolvin, J. Chem. Phys. 128, 164315 (2008).

${ }^{74}$ B. O. Roos and K. Andersson, Chem. Phys. Lett. 245, 215 (1995).

${ }^{75}$ N. Forsberg and P.-Aa. Malmqvist, Chem. Phys. Lett. 274, 196 (1997).

${ }^{76}$ K. Do, T. P. Klein, C. A. Pommerening, and L. S. Sunderlin, J. Am. Soc. Mass Spectrom. 8, 688 (1997).

${ }^{77}$ J. Gao, W. Zou, W. Liu, Y. Xiao, D. Peng, B. Song, and C. Liu, J. Chem. Phys. 123, 054102 (2005).

${ }^{78}$ K. Pierloot, E. van Besien, E. van Lenthe, and E. J. Baerends, J. Chem. Phys. 126, 194311 (2007).

${ }^{79}$ R. Bast, H. J. A. Jensen, and T. Saue, Int. J. Quantum Chem. 109, 2091 (2009).

${ }^{80}$ J. P. Perdew and A. Zunger, Phys. Rev. B 23, 5048 (1981).

${ }^{81}$ C. Angeli, R. Cimiraglia, S. Evangelisti, T. Leininger, and J. P. Malrieu, J. Chem. Phys. 114, 10252 (2001).

${ }^{82}$ P. Fuentealba, H. Preuss, H. Stoll, and L. V. Szentpály, Chem. Phys. Lett. 89, 418 (1982).

${ }^{83}$ M. Dolg, in Relativistic Electronic Structure Theory-Part 1. Fundamentals, edited by P. Schwerdtfeger (Elsevier, Amsterdam, 2002), p. 523.

${ }^{84}$ E. Fromager, J. Toulouse, and H. J. A. Jensen, J. Chem. Phys. 126, 074111 (2007). 